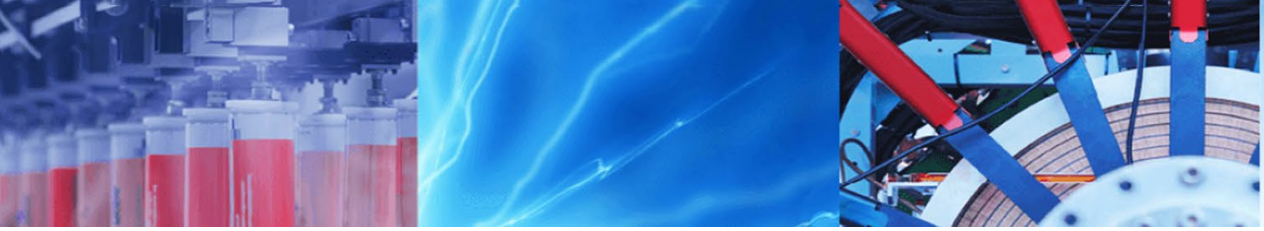

Research Article

\title{
An improved chacha algorithm for securing data on loT devices
}

\author{
Mohammed Salih Mahdi ${ }^{1}$ Nidaa Falih Hassan ${ }^{2}$. Ghassan H. Abdul-Majeed ${ }^{3}$ (1)
}

Received: 16 August 2020 / Accepted: 24 February 2021 / Published online: 6 March 2021

(c) The Author(s) 2021 OPEN

\begin{abstract}
In recent years, revolution of development was exceedingly quick in the Internet. Nevertheless, instead of only linking personal computers, mobiles and wearable equipment's, Internet growths from a web binding to true world physical substances that is indicated to novel connotation, which is labeled as Internet of Things (IoT). This concept is utilized in many scopes like education, health care, agriculture and commerce. loT devices are presented with batteries to have independence from electric current; consequently, their working time is specified by the total time of the power of these batteries. In many loT applications, data of loT devices are extremely critical and should be encrypted. Current encryption approaches are created with a high complexity of an arithmetical process to provide a high level of security. However, these arithmetical processes lead to troubles concerning the efficiency and power consumption. ChaCha cipher is one of these approaches, which recently attracted attention due to its deployment in several applications by Google. In the present study, a new stream cipher procedure is proposed (called Super ChaCha), which performs low duty cycles for securing data on loT devices. The proposed algorithm represents an improved revision to the standard ChaCha algorithm by increasing resistance to cryptanalysis. The modification focuses on rotation procedure which has been changed from a fixed constant to a variable constant based on random value. Also, the inputs of the cipher are changing in the columns form followed by diagonals form to zigzag form and then by alternate form to provide improved diffusion in comparison with the standard ChaCha. Results regarding the security illustrate that Super ChaCha needs 2512 probable keys to break by brute-force attack. Furthermore, the randomness of Super ChaCha successfully passed the five benchmark and NIST test.
\end{abstract}

Keywords Internet of Things · Power consumption · Stream cipher · ChaCha

\section{Introduction}

The expression "Internet" indicates a senior denomination of protocols and applications; it is established on best of advanced and associated networks. In 24/7 fashion, it assists zillions of humans around the continents [1]. From 1990 to the time being, revolution of development was exceedingly quick in the Internet, growths from a web environment to real-world objects that is indicated to Internet of Things (IoT) [2]. IoT is a network of physical devices that have the ability to sense and collect data from the outside environment. The loT devices can exist in different geographic locations, and the collected data can be used for different applications in areas such as health care, education, banking, and others. The loT technology is a modern standard, which is rapidly obtained scope by combining sets of models and communication. The main concept of the loT includes a diversity of objects like emergency mechanisms (Bluetooth, RFID, sensors) and mobile phones. It has eligiblity to communicate with another mechanism across the Internet. The fast improvement of IoT can help people to make their situation smarter and more computable, overcoming daily problems. In short, loT presents new opportunities to monitor and manage

Ghassan H. Abdul-Majeed, ghassan@uob.edu.iq | 'University of Information Technology and Communication, Baghdad, Iraq. ${ }^{2}$ University of Technology, Baghdad, Iraq. ${ }^{3}$ Al-Mashreq University, Baghdad, Iraq. 
devices remotely, and hence take decisions based on the information received from various real-time traffic data streams [3-5].

The loT structure consists of five components, namely sensor, aggregator, transmission channel, external utility, and decision trigger [6]. A sensor is an electronic utility for measuring physical attributes (temperature, weight, location, and others). The aggregator is a software dedicated for transforming raw data into aggregated data. The transmission channel is a medium for transmitting the collected data (USB, wireless, wired, or verbal). The external utility (which is either software or hardware) is designed for storage purpose. The decision trigger conceptually defines the end purpose of the loT. It is reasonable, in most cases, to deal with the decision trigger as an if-then rule [6].

At present, in both education and manufacturing, loT is rapidly being a major topic of the utmost technologies and trends. Furthermore, according to researchers and scientists, a switch from utilizing conventional equipment's to utilizing the Internet, is coming into a modern era to link smart equipment's [7, 8]. By 2017, forecasts illustrated that $3.6 \times 10^{9}$ of human beings linked to the Internet, sixty-four of them are linked through smartphones [9]. This guides to quicker reply times to generic emergencies, keep souls, health care, and others. Therefore, it will enhance the goodness of individuals' lifetime by presenting services via smart cities. Hence, utilize resources more expeditiously and keep the money while presenting outstanding services [10].

Generally, security problems in all scopes of loT applications are considered as the first important huge challenges [11]. Furthermore, in loT environment, every individual equipment and sensor that are established, deployed, and synchronized may be suffering from attackers at any position [12]. In many loT applications, data of node sensor are extremely critical and have to be encrypted. Current encryption procedures are associated with a large complexity of arithmetical processes to provide a high level of security. This arithmetic complexity, of course, results in consuming extra time and power. On the other hand, the sensors are suffering from limitations of storage space and power, which affect the transmission efficiency. One powerful solution to achieve high security with the abovementioned limitations is the use of lightweight encryption technology. Recently, Panagiotou et al. [3] encouraged using stream ciphers as lightweight cryptography techniques for sensitive data in loT devices.

Various suggested procedures have been implemented lightweight encryption schemes for loT. Yao et al. [13] offered a lightweight encryption procedure for securing information transport according to attribute-based encryption and Elliptic Curve Decisional Diffie Hellman. Furthermore, they suggested procedure-resisted resource-limited equipment in loT security troubles and assisted to enhance performance efficiency. Salami et al. [14] handled resource-limited equipment in a smart house of its security troubles by suggesting a lightweight encryption procedure according to identity-based (public key management) that do not require certificate for securing sending information between householder and smart things in the house. Baskar et al. [15] explained WSN concept and their components and properties and focused on WSN, where it was apt to attack. In addition, they suggested lightweight encryption procedure by employing chaos map to provide key by utilizing Field Programmable Gate Array. Yang et al. [16] suggested secure data administration of patients utilizing keyword search and lightweight encryption procedure with Diffie Hellman of health data of patients in health care scope. Recently, Panagiotou et al. [3] introduced a symmetric cryptography, based on the Advanced Encryption Standard (AES), for both encryption and decryption of texts, images and electronic data applications in loT devices. Hammi et al. [17] proposed a lightweight elliptic curve cryptography-based authentication scheme for loT. They adopted one-time-password as an authentication scheme in which a new password is generated for each authentication session and the reuse of a password is not possible. More recently, Kponyo et al. [18] have proposed a lightweight and host-based DoS anomaly detection and defense mechanism for resourceconstrained loT devices. Their approach was dedicated to address DoS attacks on loT devices.

The objective of this study is to suggest a lightweight stream cipher algorithm, with low power consumption, for securing the information that exchanged between cloudlevel (Google Cloud) as backend real-time database and loT sensors.

\section{Stream Cipher and Chacha}

From 1990 to 2020, a huge evolution occurs in the scope of data storing, telecommunication, and Internet. These modern evolutions need robust security, according to robust cryptography procedure [19]. Cryptography is a procedure for safe communication in the presence of adversaries [20]. Cryptography procedures are mostly categorized into two forms: The first is a block cipher that indicates the procedure of the cipher by splitting each original data into sequential blocks and every block is encrypted by utilizing identical key $[21,22]$. The second form is a stream cipher that indicates the procedure of the cipher by utilizing XOR function between the original data and key random series for getting the cipher data [23].

This paper concentrates only on the stream cipher due to its suitability in scopes like its equipment and mobile 
Fig. 1 Input of ChaCha20

\begin{tabular}{|c|c|c|c|}
\hline $\mathrm{c} 1$ & $\mathrm{c} 2$ & $\mathrm{c} 3$ & $\mathrm{c} 4$ \\
\hline $\mathrm{k} 1$ & $\mathrm{k} 2$ & $\mathrm{k} 3$ & $\mathrm{k} 4$ \\
\hline $\mathrm{k} 5$ & $\mathrm{k} 6$ & $\mathrm{k} 7$ & $\mathrm{k} 8$ \\
\hline $\mathrm{b} 1$ & $\mathrm{~b} 2$ & $\mathrm{n} 1$ & $\mathrm{n} 2$ \\
\hline \multicolumn{5}{|l|}{} & & \\
\hline \multicolumn{5}{|l|}{} & $\mathrm{x} 1$ & $\mathrm{x} 2$ & $\mathrm{x} 3$ \\
\hline $\mathrm{x} 0$ & $\mathrm{x} 5$ & $\mathrm{x} 6$ & $\mathrm{x} 7$ \\
\hline $\mathrm{x} 4$ & $\mathrm{x} 9$ & $\mathrm{x} 10$ & $\mathrm{x} 11$ \\
\hline $\mathrm{x} 8$ & $\mathrm{x} 13$ & $\mathrm{x} 14$ & $\mathrm{x} 15$ \\
\hline
\end{tabular}

Fig. 2 Quarter round function of ChaCha20

\begin{tabular}{|c|}
\hline Column Form \\
\hline $\mathrm{QR}(\mathrm{x} 0, \mathrm{x} 4, \mathrm{x} 8, \mathrm{x} 12)$ \\
\hline $\mathrm{QR}(\mathrm{x} 1, \mathrm{x} 5, \mathrm{x} 9, \mathrm{x} 13)$ \\
\hline $\mathrm{QR}(\mathrm{x} 2, \mathrm{x} 6, \mathrm{x} 10, \mathrm{x} 14)$ \\
\hline $\mathrm{QR}(\mathrm{x} 3, \mathrm{x} 7, \mathrm{x} 11, \mathrm{x} 15)$ \\
\hline \\
\hline Row Form \\
\hline $\mathrm{QR}(\mathrm{x} 0, \mathrm{x} 5, \mathrm{x} 10, \mathrm{x} 15)$ \\
\hline $\mathrm{QR}(\mathrm{x} 1, \mathrm{x} 6, \mathrm{x} 11, \mathrm{x} 12)$ \\
\hline $\mathrm{QR}(\mathrm{x} 2, \mathrm{x} 7, \mathrm{x} 8, \mathrm{x} 13)$ \\
\hline $\mathrm{QR}(\mathrm{x} 3, \mathrm{x} 4, \mathrm{x} 9, \mathrm{x} 14)$ \\
\hline
\end{tabular}

communications, etc. These scopes have huge resource utilization and restricted dealings with bandwidth, energy and effectively processing than block ciphers.

ChaCha20 is stream cipher utilized counter mode for symmetric encryption, ChaCha20 is implemented by Google. The total size of the input of the ChaCha20 is 512 bits, as shown in Fig. 1. These bits act as seeds, each one has 32 bits, which consist of [24]:

- 256 bit act as the total size of the key ( $k 1 \ldots k 8)$.

- 192 bit act as the total size of the nonce $(n 1, n 2)$ and constants [c1 ...c4].

- 64 bit act as the total size of the block message counter $(b 1, b 2)$.

Hence, for encryption original data utilizing ChaCha20, three lightweight procedures are used for mixing the input of the ChaCha20 to create a series of 512 bits which are symbolizing the keystream by XOR operation with the original data. The first lightweight procedure is an addition (adding of two 32 bits), the second lightweight procedure is Exclusive-OR (XORing of the two 32 bits) and the third lightweight procedure is rotation, which is in charge of rotation of 32 bits by e bit $[y \ll e]$ and $e$ acts as a constant number. The three lightweight procedures are summarized into dual function. The Quarter Round Function (QRF) represents the core

\begin{tabular}{|l|l|}
\hline $\mathrm{a}=\mathrm{a}+\mathrm{b}$ & $\longrightarrow \mathrm{d}=(\mathrm{d} \oplus \mathrm{a})<<16$ \\
\hline $\mathrm{c}=\mathrm{c}+\mathrm{d}$ & $\longrightarrow \mathrm{b}=(\mathrm{b} \oplus \mathrm{c})<<12$ \\
\hline $\mathrm{a}=\mathrm{a}+\mathrm{b}$ & $\longrightarrow \mathrm{d}=(\mathrm{d} \oplus \mathrm{a})<<8$ \\
\hline $\mathrm{c}=\mathrm{c}+\mathrm{d}$ & $\longrightarrow \mathrm{b}=(\mathrm{b} \oplus \mathrm{c})<<7$ \\
\hline
\end{tabular}

Fig. 3 Procedures in quarter round function of ChaCha20

of dual function, introduced to update the state matrix in each round. QRF is applied first on the columns and then on the diagonals of the state matrix as shown in Fig. 2 [25]:

Input values for QRF are four 32-bits and the outputs are modifying since 32-bit based on the three lightweight procedures as shown in Fig. 3.

The last process in ChaCha20 is applying an addition operation between the last modified matrix and the first seed of input ChaCha20 [26].

\section{Criteria's of Randomness of Keystream}

In this section, we will discuss two types of criteria for testing the degree of randomness of the generated binary series of the keystream generator [27]: 


\subsection{Five Basic Tests}

The performance of randomness characteristic of the keystream generator is evaluated by utilizing the five basic tests (benchmark tests) as illustrated in Table $1[28,29]$. This test examines just the output sequences of keystream generator, which is known as an empirical test [30].

\subsection{National Institute of Standards and Technology Benchmark Tests}

National Institute of Standards and Technology (NIST) Benchmark Tests encompass standardized tests which

Table 1 Benchmarked five tests equations and information [30] are utilized to check the randomness of generating binary series of the keystream generator [31, 32]. If the generated binary series pass NIST Benchmark Tests, then that series has randomness, whereas when the generated binary series fail in NIST Benchmark Tests, the series is non-random [33]. All NIST Benchmark Tests check the randomness according to parameters $(n, m, M)$ as shown in Table 2 [34]. Only NIST Benchmark Tests (bold green color) that do not exceed the bit length of the suggested keystream generator will be utilized in this paper (512 bits needed for ChaCha20 and super ChaCha).

\begin{tabular}{|c|c|}
\hline Five benchmark tests equations & Information on five benchmark tests \\
\hline$T 1=\frac{(M 0-M 1)^{2}}{M}$ & $\begin{array}{l}\text { M0: number of 0's in keystream } \\
\text { M1: number of 1's in keystream } \\
\text { M: total size of keystream }\end{array}$ \\
\hline$T 2=\frac{4}{M-1}\left((M 11)^{2}+(M 00)^{2}+(M 01)^{2}+(M 10)^{2}\right)-\frac{2}{M}\left(M 1^{2}+M 0^{2}\right)+1$ & $\begin{array}{l}\text { M11: number of } 11 \text { 's in keystream } \\
\text { M00: number of } 00 \text { 's in keystream } \\
\text { M01: number of } 01 \text { 's in keystream } \\
\text { M10: number of } 10 \text { 's in keystream }\end{array}$ \\
\hline $\begin{array}{l}P=\frac{M}{N} \quad \frac{M}{N} \geq\left(5 * 2^{N}\right) \\
T 3=\frac{2^{N}}{P}\left(\sum_{j=1}^{2^{N}} M_{j}^{2}\right)-P\end{array}$ & $M_{j}$ : number of appearance of the $j$ th of length $\mathrm{N}$ \\
\hline $\begin{array}{l}P_{j}=\frac{M-j+3}{2(j+2)} \\
T 4=\left(\sum_{j=1}^{N} \frac{\left(B_{j}-P_{j}\right)^{2}}{P_{j}}\right)+\left(\sum_{j=1}^{N} \frac{\left(G_{j}-P_{j}\right)^{2}}{P_{j}}\right) \\
A(k)=\sum_{j=0}^{M-k-1}\left(S_{j}+S_{+k}\right) \operatorname{Mod} 2 \\
T 5=\frac{2\left(A(k)-\frac{(M-k)}{2}\right)}{\sqrt{(M-k)}}\end{array}$ & $\begin{array}{l}N: \text { maximum } \mathrm{j} \text { for which } P_{j} \geq 5 \\
B_{j}: \text { Amount of blocks (subsequences runs of } 1 \text { 's) of length } j \text { in } M \\
G_{j}: \text { amount of gabs(subsequences runs of } 0 \text { 's) of length } j \text { in } M \\
k: 1 \leq k \leq[m / 2]\end{array}$ \\
\hline
\end{tabular}

Table 2 Values of parameters of NIST tests [34]

\begin{tabular}{lllll}
\hline Test\# & Test name & $n$ & $m$ or $M$ & \#Sub-test \\
\hline 1 & Frequency & $n \geq 100$ & - & 1 \\
2 & Frequency within a block & $n \geq 100$ & $20 \leq M \leq n / 100$ & 1 \\
3 & Runs & $n \geq 100$ & - & 1 \\
4 & Longest run of ones & $n \geq 128$ & & 1 \\
5 & Rank & $n \geq 38,912$ & - & 1 \\
6 & Spectral & $n \geq 1000$ & - & $148^{*}$ \\
7 & Non-overlapping T.M & $n \geq 8 m-8$ & $2 \leq m \leq 21$ & 1 \\
8 & Overlapping T.M & $n \geq 10^{6}$ & & 1 \\
9 & Maurer's universal & $n>387,840$ & & 1 \\
10 & Linear complexity & $n \geq 10^{6}$ & $500 \leq M \leq 5000$ & 2 \\
11 & Serial & & $2<m<\left[\log _{2} n\right]-2$ & 1 \\
12 & Approximate entropy & & $m<\left[\log _{2} n\right]-5$ & 2 \\
13 & Cumulative sums & $n \geq 100$ & & 8 \\
14 & Random excursions & $n \geq 10^{6}$ & & 18 \\
15 & Random excursions variant & $n \geq 10^{6}$ & & \\
\hline
\end{tabular}




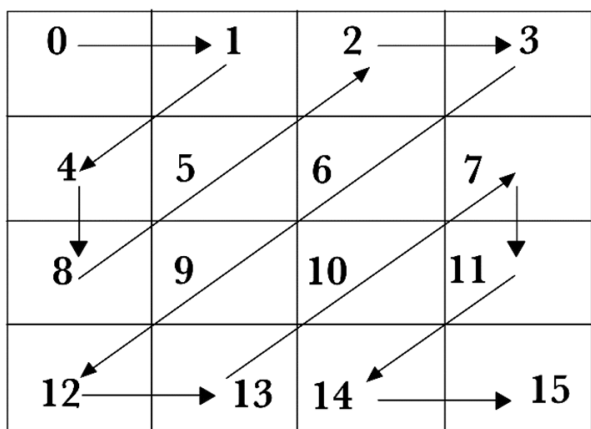

Fig. 4 Zigzag form

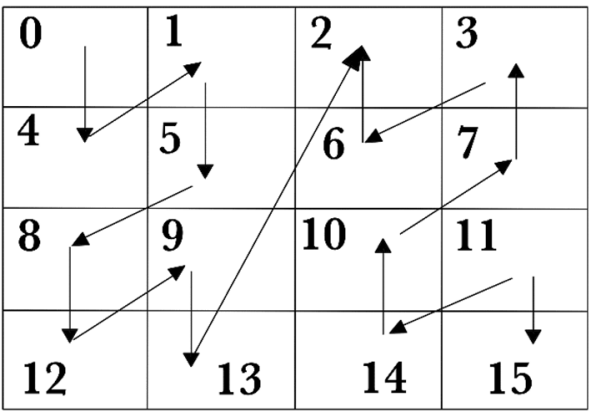

Fig. 5 Alternate form

\section{Suggested Super ChaCha}

The fundamentals of the ChaCha20 have been studied to build a new keystream generator for producing keys, taking into consideration the increasing of security level and decreasing the complicated stages. The producing keys will be used for encryption loT data. We denote the new approach as Super ChaCha Lightweight Stream Cipher, described in Algorithm 1, which consists of 10 rounds ultimately summarized into:

- The rotation procedure $(16,12,8$, and 7$)$ in ChaCha20 is modified from a fixed constant to a variable constant based on random value $(y 0, y 1, y 2$, and $y 3)$, respectively, in each round, as shown in Algorithm 2.

- The order of application of the QRF (for updating inputs) has been changed in the columns from followed by diagonals form to zigzag form and then by alternate form as shown in Figs. 4 and 5. This new order of updating process results in more diffusion of inputs and thereby increasing the complexity against attacks.
For encryption loT data sensor, an XOR operation is applied between the 512 bits of keystream, which is generated from Algorithm 1 and loT sensor.

\begin{tabular}{|c|}
\hline Algorithm 1: Super ChaCha keystream Generator \\
\hline $\begin{array}{l}\text { Input: } 512 \text { bit (seed key, nonce, counter and constants) stored } \\
\text { in a matrix } \mathrm{x}(\mathrm{x} 0, \mathrm{x} 1, \mathrm{x} 2, \ldots \ldots, \mathrm{x} 15)\end{array}$ \\
\hline Output: 512 bit (keystream) \\
\hline $\begin{array}{l}\text { Begin } \\
\text { Step1: round=1 } \\
\text { Step2: repeat } \\
\text { ChaCha-quarter function }(\mathrm{x} 0, \mathrm{x} 1, \mathrm{x} 4, \mathrm{x} 8) \\
\text { ChaCha-quarter function }(\mathrm{x} 5, \mathrm{x} 2, \mathrm{x} 3, \mathrm{x} 6) \\
\text { ChaCha-quarter function }(\mathrm{x} 9, \mathrm{x} 12, \mathrm{x} 13, \mathrm{x} 10) \\
\text { ChaCha-quarter function }(\mathrm{x} 7, \mathrm{x} 11, \mathrm{x} 14, \mathrm{x} 15) \\
\text { ChaCha-quarter function }(\mathrm{x} 0, \mathrm{x} 4 \times \mathrm{x} 1, \mathrm{x} 5) \text {, } \\
\text { ChaCha-quarter function }(\mathrm{x} 8, \mathrm{x} 12, \mathrm{x} 9, \mathrm{x} 13) \text {, } \\
\text { ChaCha-quarter function }(\mathrm{x} 2, \mathrm{x} 6, \mathrm{x} 3, \mathrm{x} 7) \text {, } \\
\text { ChaCha-quarter function }(\mathrm{x} 10, \mathrm{x} 14, \mathrm{x} 11, \mathrm{x} 15) \text {. } \\
\text { Step3: round =round }+1 \\
\text { Step4: until round less than or equal to } 10 \\
\text { Step5: store the update matrix } \mathrm{x} \text { in keystream matrix as key } \\
\text { End }\end{array}$ \\
\hline
\end{tabular}

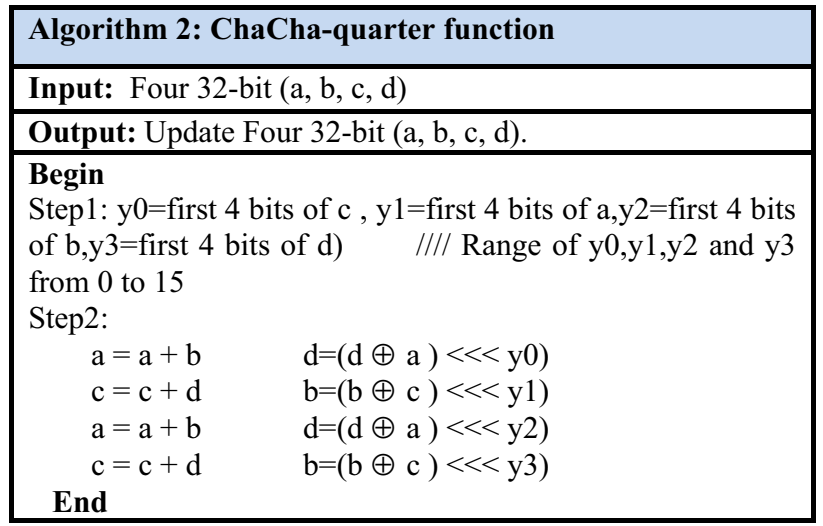

\section{Implementation of the Proposed Super ChaCha Keystream Cipher}

For implementation purposes, the pulse heart rate and body temperature of persons (IoT data sensor) are used as inputs, which will be encrypted based on the keystream of the proposed Super ChaCha (described in Algorithm 1). The outputs extracted by the Super ChaCha model are keys with a less complicated procedure and a good robustness keystream, proved by effectively surpasses the five benchmark tests as illustrated in Table 3 and NIST benchmark tests as illustrated in Table 4. Figure 6 shows the model of exchanging the encrypted messages between 
Table 3 Five Benchmark tests performance

\begin{tabular}{lccl}
\hline Five benchmark tests & Test value & Threshold & $\begin{array}{l}\text { Test } \\
\text { value }<\text { thresh- } \\
\text { old }\end{array}$ \\
\hline Frequency test $T 1$ & 0.03 & 3.841 & Pass \\
Serial test $T 2$ & 0.645 & 5.991 & Pass \\
Poker test $T 3$ & 12.933 & 14.067 & Pass \\
Runs test T4 & 4.062 & 9.487 & Pass \\
Autocorrelation $T 5$ & 1.283 & 1.96 & Pass \\
\hline
\end{tabular}

Table 4 NIST benchmark tests

\begin{tabular}{lll}
\hline NIST Test & $P$ value & $P$ value $>0.01$ \\
\hline Frequency & 0.6831 & Pass \\
Block frequency & 0.8165 & Pass \\
Runs & 0.9312 & Pass \\
Serial & 0.5482 & Pass \\
Approximate entropy & 0.7537 & Pass \\
Cumulative sums & 0.9817 & Pass \\
\hline
\end{tabular}

different sides: cloud-level, loT health sensors, clinician, and family.

Super ChaCha keystream is compared with standard ChaCha version (8), (12), and (20) according to time consuming in microseconds and complexity against attacks by applying on 512 bits, as shown in Table 5. The modification presented in this study is applied to the three versions of ChaCha as shown in Table 6. The comparison reveals the superiority of Super ChaCha over the standard ChaCha of versions 8 and 12, where the complexity against attacks has significantly increased with very little increase in time, ranging from 1 to 2 microsecond. The increase in complexity, shown by the proposed algorithm, is due to using a new order of updating process, which results in more diffusion of inputs. For the standard ChaCha20, the proposed modification does not result in a clear improvement in the complexity toward attacks.

Each suggested procedure on NodeMCU ESP8266 is executed according to various data lengths. Several performance metrics are measured:

\subsection{Memory Usage}

The needed space for compiling NodeMCU ESP8266 with Wi-Fi library was 2 KB. The executed Super ChaCha stream cipher needs only an extra 4-7 KB of space.

\subsection{Throughput}

Throughput had been taken, in accordance with encryption procedure, described in Figs. 2 and 3 for standard ChaCha20 and in Algorithms 1 and 2 for super ChaCha approach. The total time of converting original data to

Fig. 6 Model of exchanging of encrypted messages

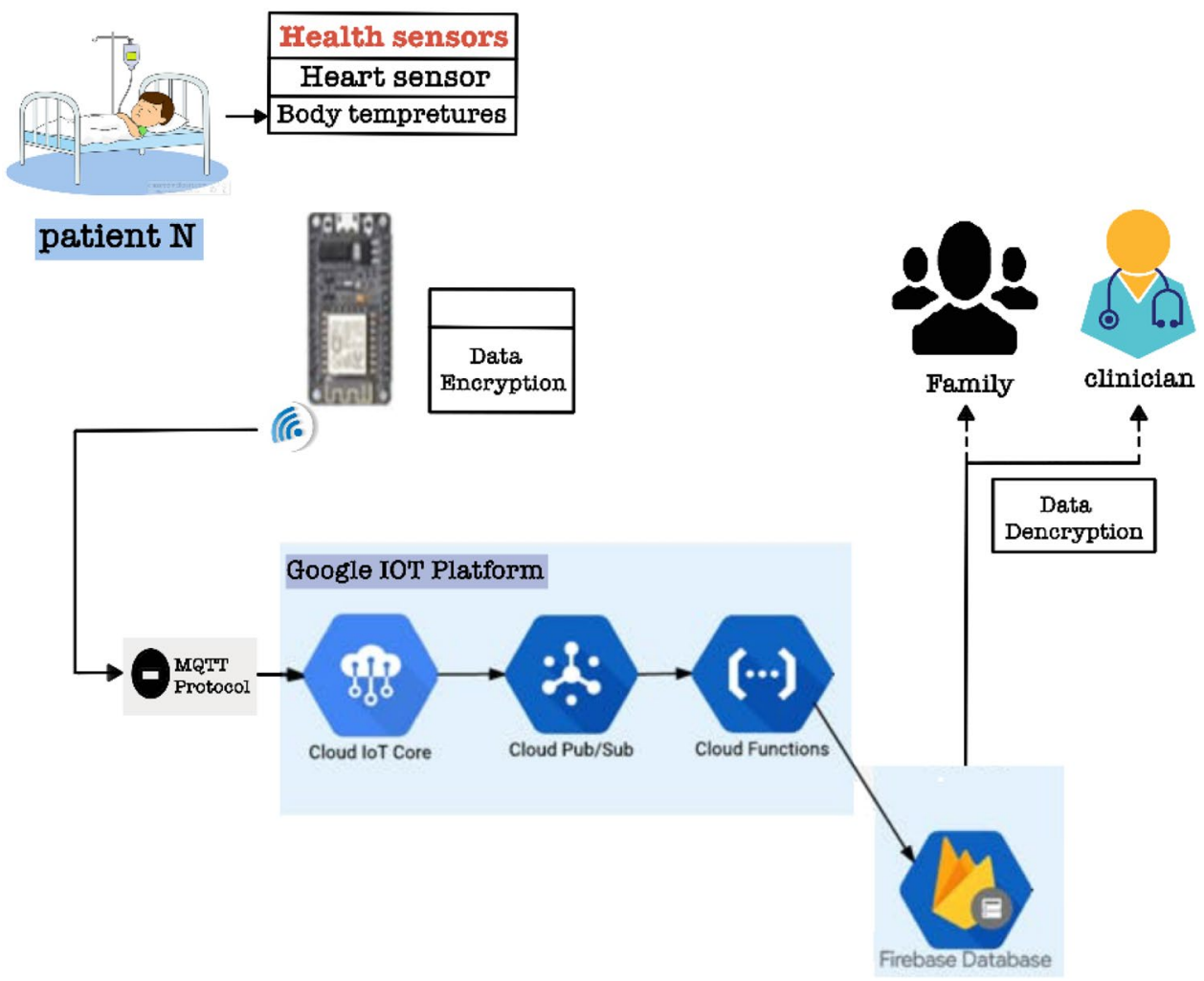


Table 5 Time consuming and complexity of standard ChaCha

\begin{tabular}{llll}
\hline Criteria & \multicolumn{3}{l}{ Standard ChaCha } \\
\cline { 2 - 4 } Versions & 8 & 12 & 20 \\
\hline Time (ms) & 19 & 23 & 28 \\
Complexity & Low & Medium & High \\
\hline
\end{tabular}

Table 6 Time consuming and complexity of super ChaCha

\begin{tabular}{llll}
\hline Criteria & \multicolumn{2}{l}{ Super ChaCha } & \\
\cline { 2 - 4 } Versions & 8 & 12 & 20 \\
\hline Time (ms) & 20 & 25 & 31 \\
Complexity & Medium & High & High \\
\hline
\end{tabular}

Table 7 Throughput of standard ChaCha

\begin{tabular}{llll}
\hline Criteria & \multicolumn{3}{l}{ Standard ChaCha } \\
\cline { 2 - 4 } Versions & 8 & 12 & 20 \\
\hline $\begin{array}{c}\text { Throughput } \\
\text { (bps) }\end{array}$ & $64 / 0.019=3368$ & $64 / 0.023=2783$ & $64 / 0.028=2286$ \\
\hline
\end{tabular}

Table 8 Throughput of super ChaCha

\begin{tabular}{|c|c|c|c|}
\hline \multirow{2}{*}{$\begin{array}{l}\text { Criteria } \\
\text { Versions }\end{array}$} & \multicolumn{3}{|l|}{ Super ChaCha } \\
\hline & 8 & 12 & 20 \\
\hline $\begin{array}{l}\text { Throughput } \\
\text { (bps) }\end{array}$ & $64 / 0.02=3200$ & $64 / 0.025=2560$ & $64 / 0.031=2065$ \\
\hline
\end{tabular}

time associated with Super ChaCha (Table 6) results in a very little decrease in throughput. It is expected that the increase in security level (illustrated in Tables 5 and 6) will lead to a decrease in throughput. It is worth noting that the significant improvement in security results in a very slight decrease in throughput.

\subsection{Power Consumption}

The power consumption, of the suggested procedures executing on NodeMCU for the first installation, is computed by the following concepts:

- Running Procedure in microseconds: It acts the entire time in microseconds that are needed for performing the suggested procedure

- Cycles of procedure: It acts the value of running a procedure in microseconds * 80 , due to the CPU clock performs at a frequency of the eighty $\mathrm{MHz}$ (eighty times each microsecond).

- As previously discussed in NodeMCU properties, the voltage is 3.3 and the current is $0.08 \mathrm{~A}$.

- Clock Cycles: It is equal to the division of 1 by frequency that is referring to $80 \times 10^{-6}$.

The measure of power consumption has been calculated in microjoules by the product of the voltage, current, cycles of procedure, and Clock Cycles as presented in Tables 9 and 10. The results show very small increase in energy consumption associated with using Super ChaCha

Table 9 Power consumption of standard ChaCha

\begin{tabular}{llll}
\hline Criteria & \multicolumn{2}{l}{ Standard ChaCha } & \\
\cline { 2 - 4 } Versions & 8 & 12 & 20 \\
\hline Energy consump- & $3.3 * 0.08 *(19,000 * 80) * 80 *$ & $3.3 * 0.08 *(23,000 * 80) * 80 *$ & $3.3 * 0.08 *(28,000 * 80) * 80 *$ \\
tion microjoules & $10^{-6}=32.1024$ & $10^{-6}=38.8608$ & $10^{-6}=47.3088$ \\
\hline
\end{tabular}

Table 10 Power consumption of super ChaCha

\begin{tabular}{|c|c|c|}
\hline Criteria & Super ChaCha & \\
\hline Versions & 12 & 20 \\
\hline $\begin{array}{l}\text { Energy consump- } \\
\text { tion microjoules }\end{array}$ & $3.3 * 0.08 *(20,000 * 80) * 80 * 10^{-6}=33.7923 .3 * 0.08 *(25,000 * 80) * 80 * 10^{-6}=42.24$ & $\begin{array}{l}3.3 * 0.08 *(31,000 * 80) * 80 * \\
10^{-6}=52.3776\end{array}$ \\
\hline
\end{tabular}

cipher data is called the total time of encryption. This time is utilized to compute the speed (throughput) of the encryption procedure by dividing the length of the original data in byte by the total time of encryption in second as shown in Tables 7 and 8 . The very slight increase in the (keeping in mind the very slight increase in time consuming for Super ChaCha, which does not exceed $3 \mathrm{~ms}$ ). In short, the clear enhancement in security causes a very small increase in energy consumption. 


\section{Conclusions}

ChaCha stream cipher had come in different versions based on number of rounds. So far, no fully successful attack on ChaCha12 and ChaCha20 has been published in the literature, but there are several successful attacks on ChaCha 6,7 , and 8 . In the present study, a new stream cipher is suggested, which represents a modification of ChaCha cipher (denoted as super ChaCha). The modification focuses on rotation procedure which is modified from a fixed constant to a variable constant based on random value. Also, the inputs of the cipher are changing in the columns from followed by diagonals form to zigzag form and then by alternate form. The randomness of the super ChaCha cipher successfully passed the five benchmark tests and NIST test. A comparison of super ChaCha with the standard versions of ChaCha $(8,12,20)$ has been implemented based on several parameters, including time, complexity, memory, throughput, and power consumption. Results reveal that the complexity level is significantly increased with very slight increase in time, memory, power consumption, and slight decrease in throughput. Regarding the security level, super ChaCha requires $2^{512}$ probable keys to break by brute-force attack, as compared to $2^{248}$ probable keys for ChaCha8. We believe that the super ChaCha cipher is suitable for security of loT devices which required high security but suffering from low energy and limited storage space.

\section{Declaration}

Conflict of interest The author(s) declare that they have no competing interests.

Open Access This article is licensed under a Creative Commons Attribution 4.0 International License, which permits use, sharing, adaptation, distribution and reproduction in any medium or format, as long as you give appropriate credit to the original author(s) and the source, provide a link to the Creative Commons licence, and indicate if changes were made. The images or other third party material in this article are included in the article's Creative Commons licence, unless indicated otherwise in a credit line to the material. If material is not included in the article's Creative Commons licence and your intended use is not permitted by statutory regulation or exceeds the permitted use, you will need to obtain permission directly from the copyright holder. To view a copy of this licence, visit http://creativecommons .org/licenses/by/4.0/.

\section{References}

1. Hafner K, Lyon M (1998) Where wizards stay up late: the origins of the Internet. Simon and Schuster. https://www.amazon.com/ Where-Wizards-Stay-Up-Late/dp/0684832674
2. Sundmaeker $H$, Guillemin $P$, Friess $P$, Woelfflé $S$ (2010) Vision and challenges for realising the Internet of things. Eurpean Research Projects, March. http://www.internet-of-things-research.eu/pdf/ loT_Clusterbook_March_2010.pdf.

3. Panagiotou P, Sklavos N, Darra E, Zaharakis ID (2020) Cryptographic system for data applications, in the context of internet of things. Microprocess Microsyst 72:102921. https://doi. org/10.1016/j.micpro.2019.102921

4. Atzori L, lera A, Morabito $G$ (2010) The internet of things: a survey. Comput Netw 54(15):2787-2805. https://doi.org/10.1016/j. comnet.2010.05.010

5. Theodorou S, Sklavos N (2019) Blockchain based security and privacy in smart cities. In: DB Rawat, K Ayhan, Z Ghafoor (eds) Chapter in the book: smart cities cybersecurity and privacy. Elsevier Press, 2019 ISBN: 9780128150320.

6. Jeffry V, Bill A, Phillip AL (2018) A closer look at the IOT "things". IEEE Comput Soc 20(30):11-15. https://doi.org/10.1109/ MITP.2018.032501741

7. World Economic Forum (2015) Industrial internet of things: unleashing the potential of connected products and services. http://www3.weforum.org/docs/WEFUSA_Industriallnternet_ Report2015.pdf.

8. Mahdi MS, Hassan NF (2018) A suggested lightweight lossless compression approach for internet of everything devices. J Univ Babylon Pure Appl Sci 26(9):107-118

9. Clarke RY (2013) Smart cities and the internet of everything: the foundation for delivering next generation citizen services. Technical reports, Alexandria, VA

10. Mahoney J, LeHong $H$ (2012) Innovation insight: the 'internet of everything' innovation will transform business. Gartner, Inc., Stamford, Connecticut, USA, Research Report 2012. https:// www.gartner.com/doc/1886915/innovation-insight-interneteverything-innovation

11. Zarpelão BB, Miani RS, Kawakani CT, de Alvarenga SC (2017) A survey of intrusion detection in Internet of Things. J Netw Comput Appl 84:25-37. https://doi.org/10.1016/j.jnca.2017.02.009

12. Giaretta A, Balasubramaniam S, Conti M (2016) Security vulnerabilities and countermeasures for target localization in bionanothings communication networks. IEEE Trans Inf Forensics Secur 11(4):665-676. https://doi.org/10.1109/TIFS.2015.25056 32

13. Yao $X$, Chen $Z$, Tian $Y$ (2015) A lightweight attribute-based encryption scheme for the internet of things. Future Gener Comput Syst 49:104-112. https://doi.org/10.1016/j.futur e.2014.10.010

14. Al Salami S, Baek J, Salah K, Damiani E (2016) Lightweight encryption for smart home. In: 201611 th International conference on availability, reliability and security (ARES), pp 382-388. https://doi.org/https://doi.org/10.1109/ARES.2016.40

15. Baskar C, Balasubramaniyan C, Manivannan D (2016) Establishment of light weight cryptography for resource constraint environment using FPGA. Procedia Comput Sci 78:165-171. https:// doi.org/10.1016/j.procs.2016.02.027

16. Yang Y, Zheng X, Tang C (2017) Lightweight distributed secure data management system for health internet of things. J Netw Comput Appl 89:26-37. https://doi.org/10.1016/j. jnca.2016.11.017

17. Hammi B, Fayad A, Khatoun R, Zeadally S, Begriche Y (2020) A lightweight ECC-based authentication scheme for Internet of Things (IoT). IEEE Syst J 14:3440-3450. https://doi.org/10.1109/ JSYST.2020.2970167

18. Kponyo JJ, Agyemang JO, Klogo GS, Boateng JO (2020) Lightweight and host-based denial of service (DoS) detection and defense mechanism for resource-constrained loT devices. Internet Things J 12:100319. https://doi.org/10.1016/j.iot.2020.10031 9 
19. Mao W (2003) Modern cryptography: theory and practice. Prentice Hall professional technical reference. https://books.googl e.iq/books/about/Modern_Cryptography.html?id=H42WQ gAACAAJ\&redir_esc $=y$

20. Mahdi MS, Hassan NF (2018) Design of keystream Generator utilizing Firefly Algorithm. J Al Qadisiyah Comput Sci Math 10(3):91. https://doi.org/10.29304/jqcm.2018.10.3.441

21. Cusick TW, Ding C, Renvall AR (2004) Stream ciphers and number theory, vol 66. Elsevier, Amsterdam

22. Bach E, Shallit JO (1996) Algorithmic number theory: efficient algorithms, vol 1. MIT Press, Cambridge

23. Minglin $Y$, Junshuang $M$ (2011) Stream ciphers on wireless sensor networks. In: Measuring technology and mechatronics automation (ICMTMA), vol 3, pp 358-361. doi: https://doi. org/10.1109/ICMTMA.2011.660

24. Yadav P, Gupta I, Murthy SK (2016) Study and analysis of eSTREAM cipher Salsa and ChaCha. In: IEEE international conference on engineering and technology (ICETECH), pp 90-94. doi: https://doi.org/10.1109/ICETECH.2016.7569218.

25. Nir Y, Langley A (2018) ChaCha20 and Poly1305 for IETF Protocols. Internet research task force, request for comments: 8439. https://datatracker.ietf.org/doc/rfc7539/

26. Bernstein DJ (2008) Chacha, a variant of ChaCha20. In: Workshop record of SASC, vol 8. http://cr.yp.to/chacha/chacha-20080120. pdf

27. Banks DL (1999) Statistical testing of random number generators. In: Proceedings of the 22 nd national information systems security conference, October 18-21. https://www.nist.gov/publi cations/statistical-testing-random-number-generators

28. Rukhin A, Soto J, Nechvatal J, Smid M, Barker E (2001) A statistical test suite for random and pseudorandom number generators for cryptographic applications. National Institute of Standards and Technology (US). https://permanent.fdlp.gov/ Ips72078/sp-800-22-051501.pdf

29. Mahdi MS (2013) Proposed block cipher algorithm with cloud computing based on keys generator. MS Thesis, University of Technology, Iraq. https://uotechnology.edu.iq/dep-cs/mypag es/library/abstracts/msc0037.pdf

30. Mahdi MS, Hassan NF (2018) A suggested super SALSA stream cipher. Iraqi J Comput Inform IJCl 44:2. https://doi.org/10.25195 /2017/4422

31. Hammood MM, Sagheer KYAM (2015) Enhancing security and speed of RC4. Int J Comput Netw Technol. https://doi. org/10.12785/IJCNT/030201

32. Soto J, Bassham L (2000) Randomness testing of the advanced encryption standard finalist candidates. Booz-Allen and Hamilton Inc, Mclean

33. Sadeeq AT, Farhan APDAK, Hassan SA (2017) A proposed public key encryption based on hybrid chaotic maps. Qalaai Zainst J 2(2):64-71. https://doi.org/10.25212/lfu.qzj.2.2.08

34. Marton K, Suciu A (2015) On the interpretation of results from the NIST statistical test suite. Romanian J Inf Sci Technol 18(1):18-32

Publisher's Note Springer Nature remains neutral with regard to jurisdictional claims in published maps and institutional affiliations. 